\title{
Efficacy of very early mobilization in patients with acute stroke: a systematic review and meta-analysis
}

\author{
Ruolan Ding, Hong Zhang \\ The Typical Ward of TCM, Hospital of Chengdu University of Traditional Chinese Medicine, Chengdu, China \\ Contributions: (I) Conception and design: Both authors; (II) Administrative support: Both authors; (III) Provision of study materials or patients: \\ Both authors; (IV) Collection and assembly of data: Both authors; (V) Data analysis and interpretation: Both authors; (VI) Manuscript writing: Both \\ authors; (VII) Final approval of manuscript: Both authors. \\ Correspondence to: Hong Zhang. The Typical Ward of TCM, Hospital of Chengdu University of Traditional Chinese Medicine, No. 39 Shi-er-qiao \\ Road, Chengdu 610072, China. Email: hj6668835@163.com.
}

\begin{abstract}
Background: The incidence of stroke is increasing year by year, which seriously affects the quality of life of patients in the later period. At present, there is no clear evidence-based medical support for very early mobilization (VEM). Therefore, this study aims to systematically evaluate the effect of VEM intervention on patients with acute stroke in order to further understand the application value of VEM.

Methods: The Cochrane Library, PubMed, Embase, Web of Science, CNKI, and other databases were searched for randomized controlled trials (RCTs) on the VEM of patients with acute stroke. The articles were screened according to the inclusion and exclusion criteria. Data was extracted and the quality of the studies was evaluated. Revman 5.3 software was used to analyze the impact of VEM on adverse events after stroke, the disability rate, bed-related complications, length of hospital stay, and ability of daily living.

Results: After screening, 8 studies reported on the efficacy of VEM intervention for stroke patients. After analysis, VEM had no significant relationship with adverse events [relative risk (RR): 1.18; 95\% confidence interval (95\% CI): 0.94-1.47], disability rates (RR: 1.05; 95\% CI: 0.95-1.15), and bed-related complications (RR: 0.84; 95\% CI: 0.66-1.06). VEM was related to length of hospital stay [mean difference (MD): -1.72 ; 95\% CI: -2.63 to -0.81 ] and ability of daily living (MD: 4.97; 95\% CI: 0.15-9.79).

Discussion: VEM can shorten the hospital stay and improve the activities of daily living of patients. Additionally, it will not increase the adverse events, bed-related complications, and disability rates of acute stroke patients, suggesting that VEM is safe and effective.
\end{abstract}

Keywords: Very early mobilization (VEM); acute stroke; recovery; rehabilitation

Submitted Sep 16, 2021. Accepted for publication Nov 12, 2021.

doi: 10.21037/apm-21-2997

View this article at: https://dx.doi.org/10.21037/apm-21-2997

\section{Introduction}

Stroke, also known as cerebrovascular accident, is a group of acute cerebrovascular diseases with a sudden onset and common features of focal neurological deficits. Whether in developing or developed countries, stroke has a serious impact on human health due to its high incidence, disability, and mortality. According to the statistical analysis of the cerebrovascular disease collaborative research group, stroke is the second cause of death worldwide (1). Therefore, stroke has been recognized as one of the leading causes of death and disability globally.

Active treatment in the specialized stroke unit has significantly improved the treatment effect and prognosis of acute stroke patients, and has greatly reduced the mortality, disability, complication rate, and long-term care needs $(2,3)$. Very early mobilization (VEM) after a stroke is currently considered to be an important part of stroke unit therapy, and may be of great significance for improving the prognosis after acute stroke. VEM is usually defined as 
intensive out-of-bed (OOB) activities within 2 days of onset, including sitting, standing, and walking (4).

Due to the overall decrease in brain plasticity during the onset of symptoms, the best period for neuron repair may be in a very narrow window after the onset of stroke. There are reports that staying in bed after a stroke may cause serious complications such as falling pneumonia and deep vein thrombosis. In addition, prolonged bed rest can also cause muscle atrophy, bone resorption, and other musculoskeletal system problems, and can even cause the immune system to decline $(5,6)$. Many researchers believe that these complications and negative effects will delay the recovery of stroke patients and increase the mortality and disability rate of patients $(5,7)$. Therefore, it is speculated that VEM should be able to prevent complications caused by fixation, reduce the disability rate, and improve the prognosis of patients (4). However, some researchers hesitate about the changes in cerebral blood flow that VEM may cause, worrying that elevated or fluctuating blood pressure may worsen the outcome of stroke, and even cause falls during VEM $(8,9)$.

From the perspective of the theoretical basis of rehabilitation treatment, brain function is plastic and reorganizable (10). Brain plasticity refers to the ability of the brain to adapt, and can modify itself in structure and function to adapt to objective reality after injury. In the past 10 years, the most noteworthy area of stroke research is the in-depth understanding of the plasticity of the brain and the important role of rehabilitation therapy in the recovery of nerve function $(3,11)$. Therefore, in the functional remodeling of the central nervous system, one of the most important external factors is functional training. Because nerve cells after damage cannot be regenerated, some nerve pathways cannot be reconnected. At this time, the recovery of function depends on the compensation function of the nervous system. However, the compensation of nerve function depends on learning and training, so rehabilitation training plays a very important role in functional recovery $(11,12)$.

Based on the above neurological recovery theory, the optimal time of activity after treatment is still unknown, and it is still unclear whether VEM can improve the outcome and prognosis of stroke. Therefore, this article uses an evidence-based method to systematically evaluate the effects of ultra-early activities applied to patients with acute stroke, aiming to provide a reference for medical staff to carry out early rehabilitation. We present the following article in accordance with the PRISMA reporting checklist (available at https://dx.doi.org/10.21037/apm-21-2997).

\section{Methods}

\section{Search strategy}

A computer search of randomized controlled trials (RCTs) on ultra-early activities of acute stroke patients was performed using databases such as the Cochrane Library, PubMed, Embase, Web of Science, CNKI, Wanfang, and Weipu. The search terms were ("acute stroke" or "stroke" or "cerebral stroke" or "cerebrovascular stroke" or "apoplexy" or "cerebrovascular apoplexy" or "cerebrovascular accident" or "brain vascular accident" or "acute cerebrovascular accident" or "cerebral infarction" or "brain infarction" or "cerebral ischemic stroke" or "cerebral hemorrhage" or "cerebral hemorrhagic stroke") and ("very early rehabilitation" or "very early mobilization" or "ultra-early mobilization" or "out-of-bed mobilization" or "out-of-bed activity" or "early mobilization" or "early activity" or "early ambulation"). The search strategy used a combination of free words and subject terms, and at the same time retrospectively included reviews and references related to this research.

\section{Inclusion criteria}

The patients were required to meet the diagnostic criteria for acute stroke and were over the age of 18. All studies were RCTs regarding the use of VEM in patients with acute stroke. The safety indicators were the case fatality rate and the incidence of adverse events (including falls, stroke progression, recurrence of stroke, intracranial hemorrhage). The effectiveness indicators were disability, post-stroke complications, such as deep vein thrombosis, pulmonary embolism, urinary tract infection, pressure ulcers, and other complications caused by long-term bed rest after stroke, as well as ability of daily living, hospital stay, and neurological deficits.

\section{Exclusion criteria}

The exclusion criteria included patients with pre-stroke disability (modified Rankin Scale score $>3$ points), progressive neurological disease, transient ischemic attack, subarachnoid hemorrhage, acute coronary syndrome, heart failure, respiratory failure, and patients requiring conservative treatment. Studies did not involve the very early activity intervention, repeated publications, and animal experiments, and the outcome indicators did not involve safety or effectiveness. 


\section{Literature screening and data extraction}

According to the inclusion and exclusion criteria, two researchers independently screened the literature. In the screening process, if the two researchers disagreed, a third researcher would make an arbitration decision. Data extraction was also performed by the two researchers according to the content of the literature, and standardized tables for independent data extraction were formulated. The extracted data included the title of the included literature, the author, the publication time, the type of disease, the basic information of the research subjects, the sample size, the follow-up time, the intervention measures, the outcome indicators, the evaluation tools, and time.

\section{Quality assessment}

The quality of the included literature was evaluated and graded according to the evaluation criteria of the Cochrane Evaluation Manual. The evaluation items included: random sequence, allocation concealment, blinding of research subjects and treatment plan implementers, blind evaluation, completeness of results data, selective reporting of research findings, and other sources of bias. If the study fully met the above standards, this indicated that the risk of various biases was low, and its quality was grade A. If the study partially met the above standards, the risk of bias was moderate, and its quality was grade B. If the study did not meet the above standards at all, the risk of bias was higher, its quality was grade $\mathrm{C}$, and it was excluded.

\section{Statistical analysis}

Revman 5.3 software was used for meta-analysis. Measurement data such as adverse event rate, disability rate, bed-related complications, activities of daily living, and length of stay in hospital. were expressed by weighted mean difference (MD) and 95\% confidence interval (95\% CI). Adverse events, disability, and stroke binary classification data such as related complications were expressed by relative risk (RR) and $95 \%$ CI. The $\mathrm{I}^{2}$ heterogeneity test was performed to determine the statistical heterogeneity between the estimated values, and a sensitivity analysis was carried out according to the Cochrane systematic review method. If there was no significant heterogeneity between studies $\left(\mathrm{P}>0.1, \mathrm{I}^{2}<50 \%\right)$, the fixed effects model was used in the meta-analysis. If there was heterogeneity between studies $\left(\mathrm{P}<0.1, \mathrm{I}^{2}>50 \%\right)$, the random effects model was used. If the heterogeneity is too obvious and the source of the heterogeneity cannot be found, a qualitative description was made.

\section{Results}

\section{Search results and study characteristics}

Through the preliminary screening of the databases, 134 duplicate articles were eliminated by the NoteExpress document manager, 1,299 records were obtained, including 806 in English and 493 in Chinese. After excluding 962 reports that cannot be retrieved, 195 usable articles are obtained. After reading and further screening, we excluded 59 articles that did not have complete data, 51 articles which were non-randomized controlled studies, 77 articles that did not meet the requirements. Finally, 8 articles were included for meta-analysis. The specific search and screening process is shown in Figure 1. The basic characteristics of the 8 articles included in the study, such as author and publication year, are shown in Table 1.

\section{Meta-analysis results}

\section{Adverse event rate}

Four articles reported on the incidence of adverse events after ultra-early activities. There was no heterogeneity between the results of the studies $\left(\mathrm{P}=0.37, \mathrm{I}^{2}=6 \%\right)$, and the fixed effects model was used for meta-analysis. The results showed: RR: 1.18, 95\% CI: 0.94-1.47, $\mathrm{P}=0.15$, suggesting that compared with conventional nursing, ultra-early activity does not increase the incidence of adverse events in patients with acute stroke, as shown in Figure 2.

\section{Disability rate}

Five articles reported on the incidence of disability in patients 3 months after stroke. There was no heterogeneity among the studies $\left(\mathrm{P}=0.20, \mathrm{I}^{2}=34 \%\right)$, and the fixed effects model was used for meta-analysis. The results showed: RR: 1.05, 95\% CI: $0.95-1.15, \mathrm{P}=0.34$, indicating that ultra-early activity cannot reduce the disability rate of patients 3 months after stroke, as shown in Figure 3.

\section{Bed-related complications}

Four articles reported on post-stroke bed rest complications. Because $\mathrm{P}=0.19$ and $\mathrm{I}^{2}=37 \%$, the fixed effects model was used for meta-analysis. The results showed: RR: 0.84, $95 \%$ CI: $0.66-1.06, \mathrm{P}=0.15$, suggesting that there is no significant relationship between ultra-early activity and the occurrence 


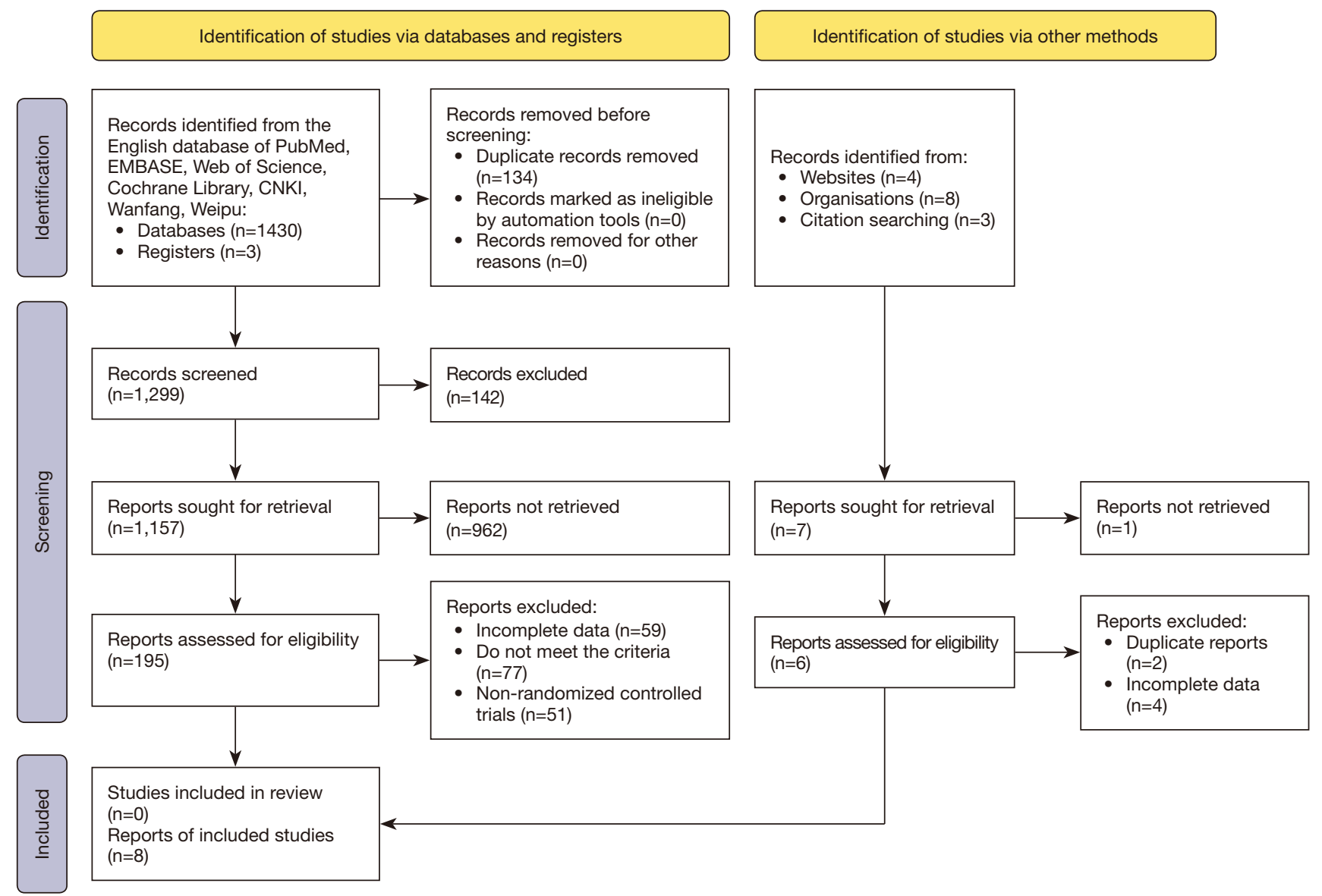

Figure 1 Flowchart of studies included in the meta-analysis.

Table 1 Basic characteristics of the study articles

\begin{tabular}{|c|c|c|c|c|c|c|}
\hline Author & Country & Year & Journal & Stroke type & \multicolumn{2}{|c|}{ Treatment measures } \\
\hline Langhorne et al. (13) & UK & 2017 & Health Technol Assess & Stroke & $\mathrm{RC}+\mathrm{VEM}$ & $\mathrm{RC}$ \\
\hline AVERT Trial Collaboration group (8) & Australia & 2015 & The Lancet & Stroke & $\mathrm{RC}+\mathrm{VEM}$ & $\mathrm{RC}$ \\
\hline van Wijk et al. (14) & Australia & 2012 & Neurorehabil Neural Repair & Stroke & VEM & $\mathrm{RC}$ \\
\hline Herisson et al. (16) & France & 2016 & PLoS One & Ischemic stroke & VEM & $\mathrm{RC}$ \\
\hline Bernhardt et al. (17) & Australia & 2008 & Stroke & Stroke & $\mathrm{RC}+\mathrm{VEM}$ & $\mathrm{RC}$ \\
\hline Sundseth et al. (9) & Norway & 2012 & Stroke & Stroke & $\mathrm{RC}+\mathrm{VEM}$ & $\mathrm{RC}$ \\
\hline Cumming et al. (18) & Australia & 2011 & Stroke & Stroke & $\mathrm{RC}+\mathrm{VEM}$ & $\mathrm{RC}$ \\
\hline
\end{tabular}

$\mathrm{RC}$, routine care; VEM, very early mobilization. 


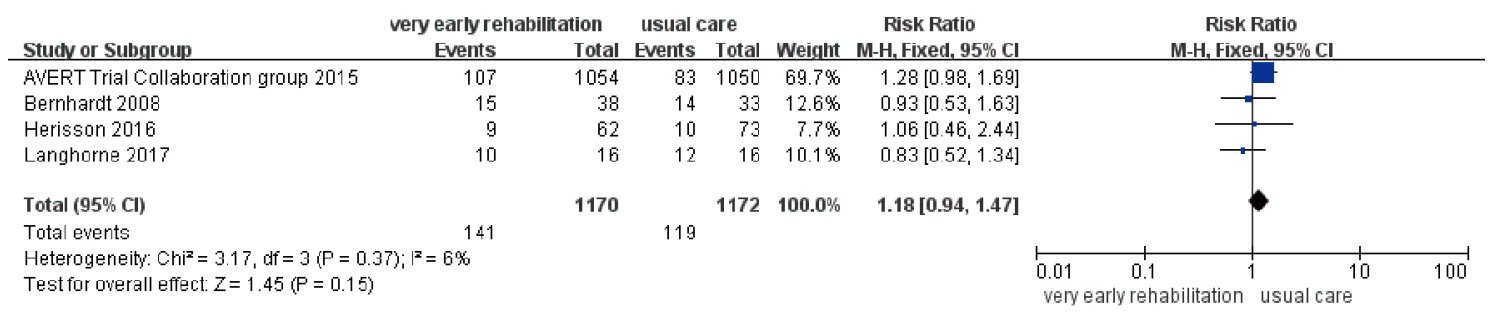

Figure 2 A meta-analysis forest plot of the incidence of adverse events using a fixed effects model. Comparison of the effects of ultra-early activities on adverse events after stroke. Statistical method: Mantel-Haenszel of fixed effects model [relative risk (RR) and 95\% confidence interval (CI)].

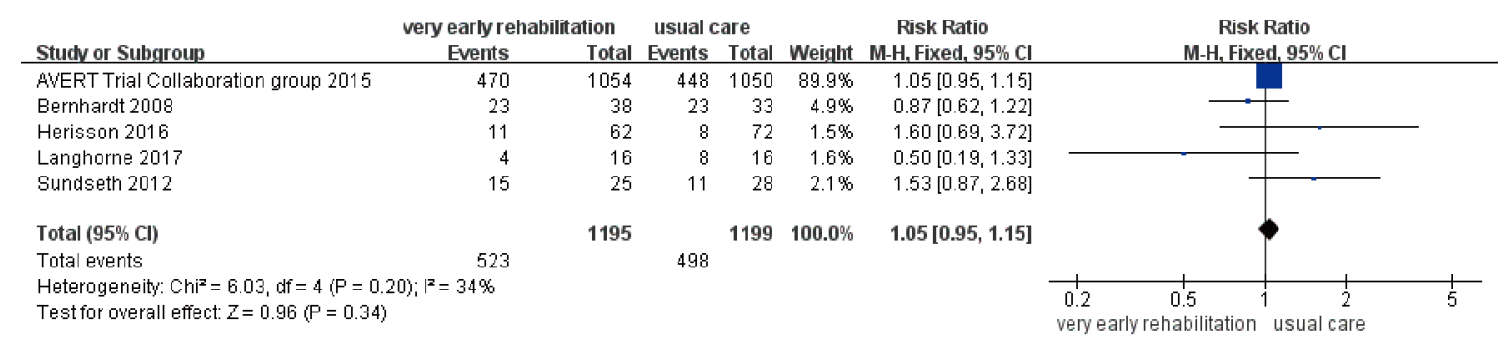

Figure 3 Forest plot of disability rate. Comparison: the effect of very early activities on the disability rate after stroke. Statistical method: Mantel-Haenszel of the fixed effects model [relative risk (RR) and 95\% confidence interval (CI)].

of complications related to bed rest after stroke, as shown in Figure 4.

\section{Hospital stay}

Four papers reported on the length of hospital stay. The analysis showed that $\mathrm{P}=0.74$ and $\mathrm{I}^{2}=0 \%$, and the fixed effects model was used for meta-analysis. The results showed: MD: $-1.72,95 \%$ CI: $-2.63--0.81, \mathrm{P}<0.001$, suggesting that ultraearly activities can shorten the hospital stay of patients with acute stroke, as shown in Figure 5.

\section{Ability of daily living}

Five articles reported on the activities of daily living of patients after stroke. The studies were heterogeneous $\left(\mathrm{P}=0.005, \mathrm{I}^{2}=73 \%\right)$, but with no clinical heterogeneity. Metaanalysis was carried out using the random effects model and the results showed: $\mathrm{MD}: 4.97,95 \% \mathrm{CI}: 0.15-9.79, \mathrm{P}=0.04$ ), suggesting that ultra-early activity can improve the activities of daily living of stroke patients, as shown in Figure 6.

\section{Publication bias}

Funnel plots were used to examine the publication bias of adverse events and hospital stay. Both funnel plots showed asymmetry, indicating that there might be publication bias, as shown in Figure 7.

\section{Risk of bias}

Among the eligible studies, all articles described that the random sequence generation was low risk $(8,9,13-18)$. Seven studies had a low risk of allocation concealment bias (9,13-18), four trials blinded participants and researchers with a low risk of bias $(14,16-18)$, and one trial had a higher risk of bias for blinding participants and researchers (16). All trials described the risk of bias in blinded results assessment [low risk $=7(8,9,13-15,17,18)$, high risk $=1(16)]$. The seven trials had a low risk of bias for incomplete outcome data $(8,9,13-15,17,18)$, and one trial had a higher risk of bias for incomplete outcome data (16). For selective reporting, all studies were judged to have a low risk of bias $(8,9,13-18)$.

\section{Discussion}

Undergoing VEM in the acute phase poses a potential risk to patients. As the body position changes during activity, the brain blood perfusion decreases when the patient's head is raised, which may aggravate neurological impairment. In addition, the increase in blood pressure caused by VEM may lead to adverse events, such as falls and increased nerve 


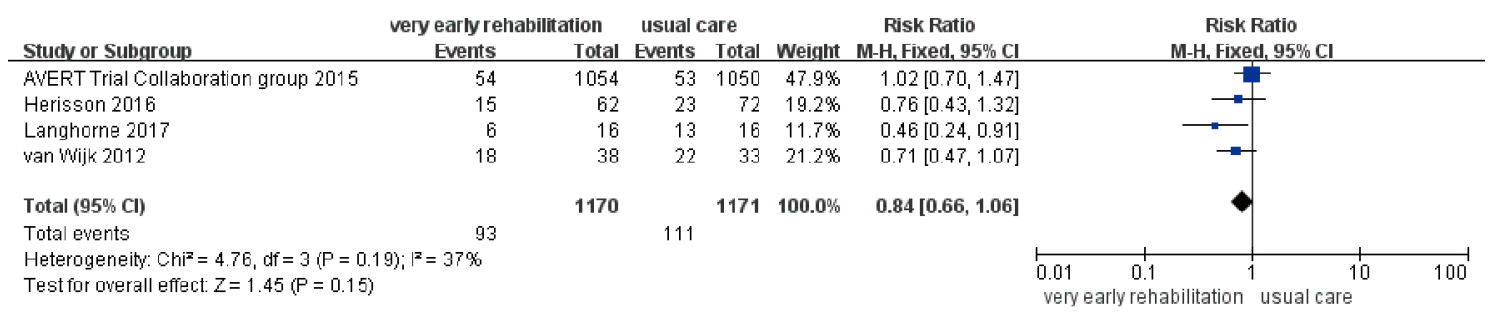

Figure 4 Forest plot of complications related to bed rest after stroke. Comparison: the impact of very early activities after stroke on bedrelated complications. Statistical method: Mantel-Haenszel of fixed effects model [relative risk (RR) and 95\% CI confidence interval].

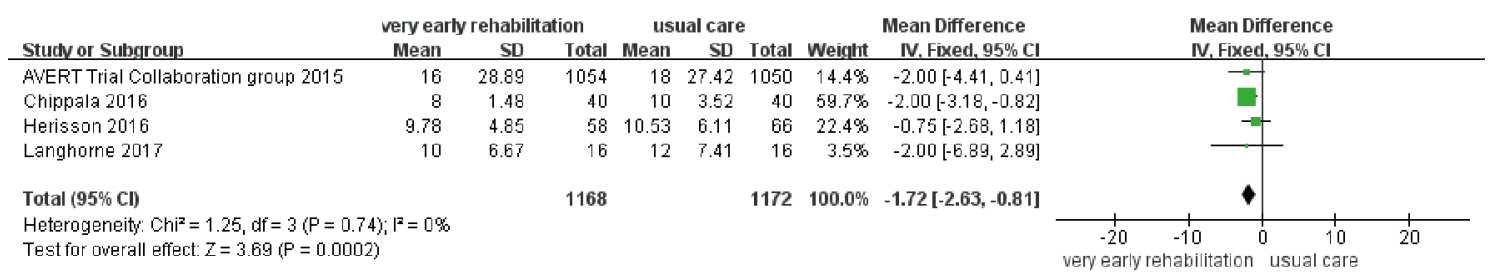

Figure 5 Forest plot of hospital stay after stroke. Comparison: the impact of ultra-early activity after stroke on length of stay in hospital. Statistical method: inverse variance of the fixed effects model [mean difference (MD) and $95 \%$ CI confidence interval].

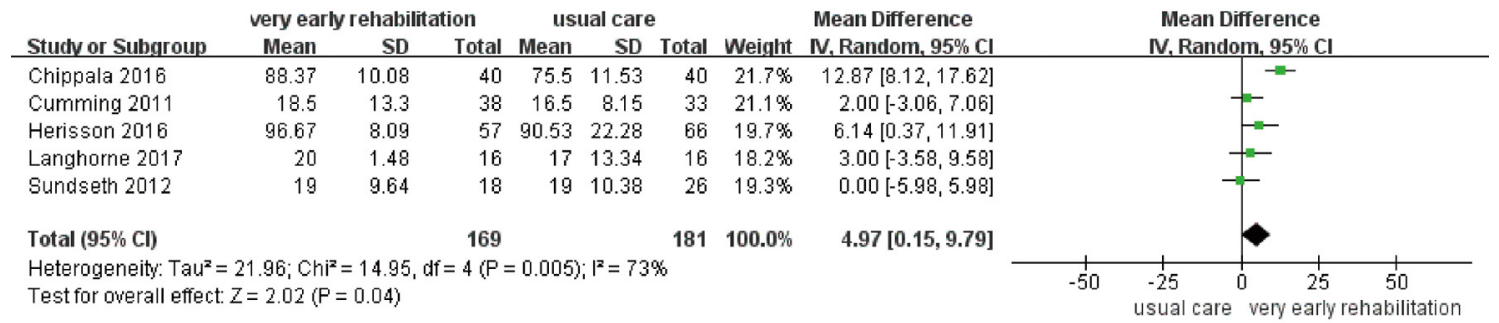

Figure 6 Forest plot of daily activity ability after stroke. Comparison: the effect of ultra-early activities after stroke on daily activity ability. Statistical method: inverse variance of random effects model [mean difference (MD) and 95\% CI confidence interval].

A

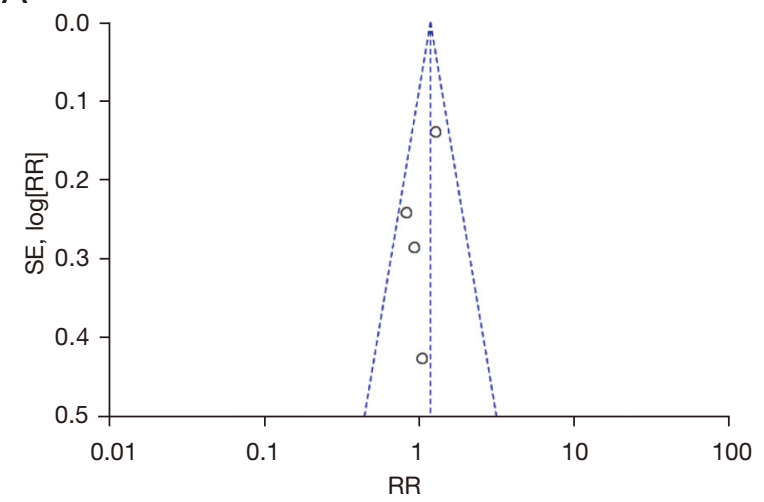

B

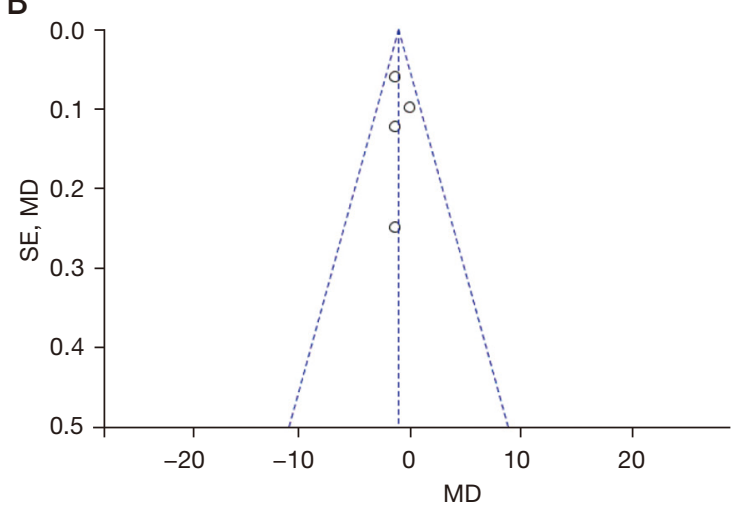

Figure 7 Funnel plot analysis of possible publication bias in subgroups. Adverse event (A) and hospital stay (B) funnel plots. MD, mean difference; SE, standard error of the mean. 
damage, which will affect the prognosis of patients $(19,20)$. The results of the meta-analysis in this study showed that VEM did not increase the incidence of adverse events in patients. The reason for this finding may be related to the better feasibility of the intervention program included in the study: when the subjects were included, their physiological indicators (systolic blood pressure $120-180 \mathrm{mmHg}$, heart rate $50-120$ beats/min, oxygen saturation $>95 \%$, body temperature $\left.<38.5^{\circ} \mathrm{C}\right)$ were limited $(14,15,17)$, which is in line with the 2017 "Chinese Stroke Early Rehabilitation Treatment Guidelines" recommending "after the patient's vital signs are stabilized, as soon as possible to carry out rehabilitation training". Before carry out VEM, monitoring the patient's standing blood pressure to be within the normal range, and the reduction should not exceed $30 \mathrm{mmHg}$ compared with the lying position $(8,14,17)$, can implement VEM, and train physical therapists or nurses on intervention content as required $(8,13,14)$, ensure the reliability and standardization of patients' brain blood perfusion and intervention.

After a stroke occurs, the expression of pro-inflammatory factors such as IL- $1 \beta$ and TNF- $\alpha$ increases within a short period of time, and the blood-brain barrier is compromised, which triggers an immune inflammatory response and neuronal apoptosis in the ischemic area, which in turn leads to aggravation of brain tissue damage. VEM can reduce blood-brain barrier damage, inhibit neuronal apoptosis, and promote angiogenesis and cerebrovascular supply by downregulating the pro-inflammatory factor IL-18 and regulating apoptosis-related proteins (21), thereby reducing brain injury. Acute stroke patients stay in bed for too long, and their daily bed time can be more than $50 \%$. Within 30 days after acute ischemic stroke, more than $51 \%$ of deaths are caused by complications of immobilization $(22,23)$. Therefore, it is recommended that patients with acute stroke undergo VEM to reduce a series of complications caused by long-term bed rest after stroke, such as pressure sores, lung infections, deep vein thrombosis, and limb contractures. The results of this study show that VEM does not reduce immobilizationrelated complications, but can improve the activities of daily living of stroke patients and shorten the hospital stay. However, it has no statistical significance in improving the disability of patients. This may be related to the inconsistency of the duration and amount of activity in the very early period. In addition, the high heterogeneity of the combined results of activities of daily living may be related to the quality of the four included articles $(9,13,15,16)$ being grade $\mathrm{B}$, and there is no impact of the age of the included subjects and the type of stroke. Subdividing the severity of stroke may cause a certain bias in the results.

Due to the complexity and uncertainty of the degree of neuroplasticity after stroke, the current definition of VEM for stroke patients can be said to be too simplistic. The present meta-analysis and included RCTs compared the efficacy and safety of the VEM group and the routine care group after a stroke, and discussed whether starting activities within 24 or 48 hours was associated with good stroke outcome $(24,25)$. VEM does not increase the risk of adverse events and cannot effectively reduce the disability rate and bed-related complications, but it can shorten the patient's hospital stay and improve the patient's ability of daily living. However, these studies did not explore the dose-response relationship between the onset of stroke patients and the degree of functional recovery. In addition, the start time and the daily frequency and duration of patient activity may be important for stroke, and further research is needed (26). Therefore, we recommend that patients with acute stroke can be given VEM if they can get out of bed early. If the patient is unwilling to exercise and is more frail, there is no need to urge the patient to exercise early to increase the patient's pain, and exercise after the patient recovers to achieve personalized treatment.

This meta-analysis has several limitations, which should be considered in the analysis. First, with the exception of the RCT (AVERT, Phase III, 2015) (8) conducted by Bernhardt and colleagues, the sample size of other articles was relatively small, which may affect the reliability of the results. Therefore, a random effects model was used to combine estimation and sensitivity analysis to reduce its influence on the results of the meta-analysis. Sensitivity analysis showed that even if Bernhardt et al.'s [2015] RCT was excluded, the aggregated results of the meta-analysis did not change significantly. Second, the meta-analysis included only 9 studies, which may reduce the strength of our results. Third, the endpoints of RCTs included were mainly evaluated 3 months after the onset of stroke, so it is impossible to analyze the dynamic changes of the efficacy and safety of VEM in stroke patients. Fourth, not all included studies assessed effectiveness and safety based on gender, therefore, we cannot assess gender differences. Fifth, all included studies enrolled stroke patients over 18 years of age, which did not allow us to analyze the effectiveness and safety of VEM in strokes in children and adolescents. Finally, the publication bias in the study should also be taken into consideration, which may have a certain impact on the results of the analysis. 


\section{Conclusions}

In summary, this study shows that ultra-early activities can improve patients' activities of daily living, and does not increase the adverse events, bed-related complications, and disability rate of patients with acute stroke. It can also shorten the hospital stay and may improve the prognosis of patients. These findings indicate that VEM is safe and effective. However, whether VEM can improve patients' neurological deficits and quality of life needs to be verified by a large sample and high-quality RCT. At the same time, future RCTs related to VEM of acute stroke are needed to subdivide the age, gender, stroke type, severity, duration of intervention, and dose of the included subjects to determine the best intervention population for VEM, to better bring health guidance evidence to patients.

\section{Acknowledgments}

Funding: This work was supported by Sichuan Province Science and Technology Planning Project (2019YFS0082) and Sichuan Provincial Cadre Health Research Project (Chuan Gan Research 2021-513).

\section{Footnote}

Reporting Checklist: The authors have completed the PRISMA reporting checklist. Available at https://dx.doi. org/10.21037/apm-21-2997

Conflicts of Interest: Both authors have completed the ICMJE uniform disclosure form (available at https://dx.doi. org/10.21037/apm-21-2997). The authors have no conflicts of interest to declare.

Ethical Statement: The authors are accountable for all aspects of the work in ensuring that questions related to the accuracy or integrity of any part of the work are appropriately investigated and resolved.

Open Access Statement: This is an Open Access article distributed in accordance with the Creative Commons Attribution-NonCommercial-NoDerivs 4.0 International License (CC BY-NC-ND 4.0), which permits the noncommercial replication and distribution of the article with the strict proviso that no changes or edits are made and the original work is properly cited (including links to both the formal publication through the relevant DOI and the license).
See: https://creativecommons.org/licenses/by-nc-nd/4.0/.

\section{References}

1. O'Donnell MJ, Chin SL, Rangarajan S, et al. Global and regional effects of potentially modifiable risk factors associated with acute stroke in 32 countries (INTERSTROKE): a case-control study. Lancet 2016;388:761-75.

2. Langhorne $\mathrm{P}$, Fearon $\mathrm{P}$, Ronning $\mathrm{OM}$, et al. Stroke unit care benefits patients with intracerebral hemorrhage: systematic review and meta-analysis. Stroke 2013;44:3044-9.

3. Langhorne P, de Villiers L, Pandian JD. Applicability of stroke-unit care to low-income and middle-income countries. Lancet Neurol 2012;11:341-8.

4. Bernhardt J, English C, Johnson L, et al. Early mobilization after stroke: early adoption but limited evidence. Stroke 2015;46:1141-6.

5. McLean DE. Medical complications experienced by a cohort of stroke survivors during inpatient, tertiarylevel stroke rehabilitation. Arch Phys Med Rehabil 2004;85:466-9.

6. Parry SM, Puthucheary ZA. The impact of extended bed rest on the musculoskeletal system in the critical care environment. Extrem Physiol Med 2015;4:16.

7. Rethnam V, Langhorne P, Churilov L, et al. Early mobilisation post-stroke: a systematic review and metaanalysis of individual participant data. Disabil Rehabil 2020. [Epub ahead of print].

8. AVERT Trial Collaboration group. Efficacy and safety of very early mobilisation within $24 \mathrm{~h}$ of stroke onset (AVERT): a randomised controlled trial. Lancet 2015;386:46-55.

9. Sundseth A, Thommessen B, Rønning OM. Outcome after mobilization within 24 hours of acute stroke: a randomized controlled trial. Stroke 2012;43:2389-94.

10. Silva GS, Nogueira RG. Endovascular Treatment of Acute Ischemic Stroke. Continuum (Minneap Minn) 2020;26:310-31.

11. Langhorne P, Collier JM, Bate PJ, et al. Very early versus delayed mobilisation after stroke. Cochrane Database Syst Rev 2018;10:CD006187.

12. Li Z, Zhang X, Wang K, et al. Effects of Early Mobilization after Acute Stroke: A Meta-Analysis of Randomized Control Trials. J Stroke Cerebrovasc Dis 2018;27:1326-37.

13. Langhorne P, Wu O, Rodgers H, et al. A Very Early Rehabilitation Trial after stroke (AVERT): a Phase III, 
multicentre, randomised controlled trial. Health Technol Assess 2017;21:1-120.

14. van Wijk R, Cumming T, Churilov L, et al. An early mobilization protocol successfully delivers more and earlier therapy to acute stroke patients: further results from phase II of AVERT. Neurorehabil Neural Repair 2012;26:20-6.

15. Chippala P, Sharma R. Effect of very early mobilisation on functional status in patients with acute stroke: a single-blind, randomized controlled trail. Clin Rehabil 2016;30:669-75.

16. Herisson F, Godard S, Volteau C, et al. Early Sitting in Ischemic Stroke Patients (SEVEL): A Randomized Controlled Trial. PLoS One 2016;11:e0149466.

17. Bernhardt J, Dewey H, Thrift A, et al. A very early rehabilitation trial for stroke (AVERT): phase II safety and feasibility. Stroke 2008;39:390-6.

18. Cumming TB, Thrift AG, Collier JM, et al. Very early mobilization after stroke fast-tracks return to walking: further results from the phase II AVERT randomized controlled trial. Stroke 2011;42:153-8.

19. Olavarría VV, Arima H, Anderson CS, et al. Head position and cerebral blood flow velocity in acute ischemic stroke: a systematic review and meta-analysis. Cerebrovasc Dis 2014;37:401-8.

20. Duss SB, Brill AK, Baillieul S, et al. Effect of early sleep apnoea treatment with adaptive servo-ventilation in acute stroke patients on cerebral lesion evolution and

Cite this article as: Ding R, Zhang H. Efficacy of very early mobilization in patients with acute stroke: a systematic review and meta-analysis. Ann Palliat Med 2021;10(11):11776-11784. doi: 10.21037/apm-21-2997 neurological outcomes: study protocol for a multicentre, randomized controlled, rater-blinded, clinical trial (eSATIS: early Sleep Apnoea Treatment in Stroke). Trials 2021;22:83.

21. Zhang P, Yu H, Zhou N, et al. Early exercise improves cerebral blood flow through increased angiogenesis in experimental stroke rat model. J Neuroeng Rehabil 2013;10:43.

22. Bernhardt J, Dewey H, Thrift A, et al. Inactive and alone: physical activity within the first 14 days of acute stroke unit care. Stroke 2004;35:1005-9.

23. Bamford J, Dennis M, Sandercock P, et al. The frequency, causes and timing of death within 30 days of a first stroke: the Oxfordshire Community Stroke Project. J Neurol Neurosurg Psychiatry 1990;53:824-9.

24. Lim BL, Lee WF, Ng WM, et al. Benefits and safety of transdermal glyceryl trinitrate in acute stroke: a systematic review and meta-analysis of randomised trials (protocol). BMJ Open 2021;11:e043591.

25. Jaworek T, Ryan KA, Gaynor BJ, et al. Exome Array Analysis of Early-Onset Ischemic Stroke. Stroke 2020;51:3356-60.

26. Xu T, Yu X, Ou S, et al. Efficacy and Safety of Very Early Mobilization in Patients with Acute Stroke: A Systematic Review and Meta-analysis. Sci Rep 2017;7:6550.

(English Language Editor: C. Betlazar-Maseh) 